\title{
A Universal Genus-Two Curve from Siegel Modular Forms
}

\author{
Andreas MALMENDIER ${ }^{\dagger}$ and Tony SHASKA ‡ \\ $\dagger$ Department of Mathematics and Statistics, Utah State University, Logan, UT 84322, USA \\ E-mail: andreas.malmendier@usu.edu \\ $\ddagger$ Department of Mathematics and Statistics, Oakland University, Rochester, MI 48309, USA \\ E-mail:shaska@oakland.edu
}

Received July 18, 2017, in final form November 25, 2017; Published online November 30, 2017

https://doi.org/10.3842/SIGMA.2017.089

\begin{abstract}
Let $\mathfrak{p}$ be any point in the moduli space of genus-two curves $\mathcal{M}_{2}$ and $K$ its field of moduli. We provide a universal equation of a genus-two curve $\mathcal{C}_{\alpha, \beta}$ defined over $K(\alpha, \beta)$, corresponding to $\mathfrak{p}$, where $\alpha$ and $\beta$ satisfy a quadratic $\alpha^{2}+b \beta^{2}=c$ such that $b$ and $c$ are given in terms of ratios of Siegel modular forms. The curve $\mathcal{C}_{\alpha, \beta}$ is defined over the field of moduli $K$ if and only if the quadratic has a $K$-rational point $(\alpha, \beta)$. We discover some interesting symmetries of the Weierstrass equation of $\mathcal{C}_{\alpha, \beta}$. This extends previous work of Mestre and others.
\end{abstract}

Key words: genus-two curves; Siegel modular forms

2010 Mathematics Subject Classification: 14H10; 14H45

\section{Introduction}

Let $\mathcal{M}_{2}$ be the moduli space of genus-two curves. It is the coarse moduli space for smooth, complete, connected curves of genus two over $\mathbb{C}$. Let $\mathfrak{p} \in \mathcal{M}_{2}(K)$, where $K$ is the field of definition of $\mathfrak{p}$. Construction a genus 2 curve $C$ corresponding to $\mathfrak{p}$ is interesting from many points of view. Mestre [18] has shown how to construct equations for genus-two curves with automorphism group of order two and defined over $\mathbb{Q}$. Mestre's work has recently received new attention from researchers in experimental number theory. For instance, in [3] a database of geometric and arithmetic invariants of genus-two curves defined over $\mathbb{Q}$ of small discriminant. In [1], the authors count the points in $\mathcal{M}_{2}(\mathbb{Q})$ according to their moduli height and create a database of genus-two curves from the moduli points in $\mathcal{M}_{2}(\mathbb{Q})$. In creating the database the main problem was that of constructing an equation for obstruction moduli points. This paper provides an equation over a minimal field of definition for any point $\mathfrak{p} \in \mathcal{M}_{2}$. Our work is therefore complimentary to the problem of finding an efficient construction for genustwo curves over finite fields with a prescribed number of rational points and the associated complexity analysis in $[4,7]$. Our equation for a genus-two curve is universal in the sense that it works for every moduli point given in terms of Igusa invariants or Siegel modular forms. It does not rely on special CM values for Siegel modular functions where the associated abelian surface has extra endomorphisms or the special invariants that can be used in theses cases (cf. [15]).

The natural question is if there exists a universal curve for the genus-two curve given in terms of a generic moduli point $\mathfrak{p} \in \mathcal{M}_{2}$. In other words, given an affine moduli point $\mathfrak{p}=(x, y, z)$, where $x, y, z$ are transcendentals, can we construct a curve corresponding to $\mathfrak{p}$ ? The answer is negative in the strict definition of "universal curve"; see [9, p. 39] for details. As we will show,

This paper is a contribution to the Special Issue on Modular Forms and String Theory in honor of Noriko Yui. The full collection is available at http://www.emis.de/journals/SIGMA/modular-forms.html 
there is a satisfactory answer in the sense that our "universal equation" applies to every moduli point $\mathfrak{p} \in \mathcal{M}_{2}$. However, the equation is often defined only over a quadratic extension of the field of moduli.

We focus mainly on constructing a genus-two curve $\mathcal{C}$ for any given point $\mathfrak{p}=\left(\mathbf{x}_{1}, \mathbf{x}_{2}, \mathbf{x}_{3}\right) \in$ $\mathcal{M}_{2}$, defined over a minimal field of definition, where $\mathbf{x}_{1}, \mathbf{x}_{2}, \mathbf{x}_{3}$ are ratios of modular forms as defined by Igusa in [11]. Our main result is as follows: For every point $\mathfrak{p} \in \mathcal{M}_{2}$ such that $\mathfrak{p} \in \mathcal{M}_{2}(K)$, where $K$ is the field of moduli, there is a genus-two curve $\mathcal{C}_{(\alpha, \beta)}$ given by

$$
\mathcal{C}_{(\alpha, \beta)}: y^{2}=\sum_{i=0}^{6} a_{i}(\alpha, \beta) x^{i},
$$

corresponding to $\mathfrak{p}$ with coefficients given by equation (3.9). This curve is defined over the field of moduli $K$ if and only if there exists a $K$-rational solution $(\alpha, \beta)$ to the quadratic

$$
\alpha^{2}+b \cdot \beta^{2}=c
$$

where $b$ and $c$ are given in terms of the moduli point $\mathfrak{p}$. There are some interesting properties of the coefficients defining $\mathcal{C}_{(\alpha, \beta)}$ which seem to be particular to this model and not noticed before.

It must be noticed that this equation is universal in the sense that it works for every moduli point $\left[J_{2}: J_{4}: J_{6}: J_{10}\right]$ given in terms of the Igusa invariants $J_{2}, J_{4}, J_{6}, J_{10}$. The equation is defined at worst over a quadratic extension of the field of moduli $K$. If the equation over the field of moduli is needed, then we must search locally for a rational point in the above quadratic when evaluated at the given $\mathfrak{p}$. In the process we discover some interesting absolute invariants (cf. equation (2.5)) which as far as we are aware have not been used before.

The paper is organized as follows: In Section 2 we give a brief summary of Siegel modular forms, classical invariants of binary sextics and the relations among them. While this material can be found in many places in the literature, there is plenty of confusion on the labeling and normalization of such invariants and relations among them. We also introduce a set of absolute invariants that is well-suited for the construction of a universal sextic.

In Section 3 we construct the equation of the genus-two curve by determining the Clebsch conic and the cubic. We diagonalize the corresponding conic and discover a new set of invariants which make the equation of this conic short and elegant. The diagonalized conic can be quickly determined from the invariants of the curve. The intersection of this conic and the cubic gives the equation of the genus-two curve. This equation shows some interesting symmetries of the coefficients, which to the knowledge of the authors have never been discovered before. When this universal equation is restricted to loci of curves with automorphisms or the Clebsch invariant $D=0$ (not covered by Mestre's approach) it shows that the field of moduli is a field of definition, results which agree with previous results of other authors.

\section{Preliminaries}

\subsection{The Siegel modular three-fold}

The Siegel three-fold is a quasi-projective variety of dimension 3 obtained from the Siegel upper half-plane of degree two which by definition is the set of two-by-two symmetric matrices over $\mathbb{C}$ whose imaginary part is positive definite, i.e.,

$$
\mathbb{H}_{2}=\left\{\underline{\tau}=\left(\begin{array}{cc}
\tau_{1} & z \\
z & \tau_{2}
\end{array}\right) \mid \tau_{1}, \tau_{2}, z \in \mathbb{C}, \operatorname{Im}\left(\tau_{1}\right) \operatorname{Im}\left(\tau_{2}\right)>\operatorname{Im}(z)^{2}, \operatorname{Im}\left(\tau_{2}\right)>0\right\},
$$

quotiented out by the action of the modular transformations $\Gamma_{2}:=\operatorname{Sp}_{4}(\mathbb{Z})$, i.e.,

$$
\mathcal{A}_{2}=\mathbb{H}_{2} / \Gamma_{2} \text {. }
$$


Each $\underline{\tau} \in \mathbb{H}_{2}$ determines a principally polarized complex abelian surface

$$
\mathbf{A}_{\underline{\tau}}=\mathbb{C}^{2} /\left\langle\mathbb{Z}^{2} \oplus \underline{\tau} \mathbb{Z}^{2}\right\rangle
$$

with period matrix $\left(\underline{\tau}, \mathbb{I}_{2}\right) \in \operatorname{Mat}(2,4 ; \mathbb{C})$. Two abelian surfaces $\mathbf{A}_{\underline{\tau}}$ and $\mathbf{A}_{\underline{\tau}^{\prime}}$ are isomorphic if and only if there is a symplectic matrix

$$
M=\left(\begin{array}{ll}
A & B \\
C & D
\end{array}\right) \in \Gamma_{2},
$$

such that $\underline{\tau}^{\prime}=M(\underline{\tau}):=(A \underline{\tau}+B)(C \underline{\tau}+D)^{-1}$. It follows that the Siegel three-fold $\mathcal{A}_{2}$ is also the set of isomorphism classes of principally polarized abelian surfaces. The sets of abelian surfaces that have the same endomorphism ring form sub-varieties of $\mathcal{A}_{2}$. The endomorphism ring of principally polarized abelian surface tensored with $\mathbb{Q}$ is either a quartic CM field, an indefinite quaternion algebra, a real quadratic field or in the generic case $\mathbb{Q}$. Irreducible components of the corresponding subsets in $\mathcal{A}_{2}$ have dimensions 0,1,2 and are known as CM points, Shimura curves and Humbert surfaces, respectively.

The Humbert surface $H_{\Delta}$ with invariant $\Delta$ is the space of principally polarized abelian surfaces admitting a symmetric endomorphism with discriminant $\Delta$. It turns out that $\Delta$ is a positive integer $\equiv 0,1 \bmod 4$. In fact, $H_{\Delta}$ is the image inside $\mathcal{A}_{2}$ under the projection of the rational divisor associated to the equation

$$
a \tau_{1}+b z+c \tau_{3}+d\left(z^{2}-\tau_{1} \tau_{2}\right)+e=0,
$$

with integers $a, b, c, d$, e satisfying $\Delta=b^{2}-4 a c-4 d e$ and $\underline{\tau}=\left(\begin{array}{cc}\tau_{1} & z \\ z & \tau_{2}\end{array}\right) \in \mathbb{H}_{2}$. For example, inside of $\mathcal{A}_{2}$ sit the Humbert surfaces $H_{1}$ and $H_{4}$ that are defined as the images under the projection of the rational divisor associated to $z=0$ and $\tau_{1}=\tau_{2}$, respectively. In fact, the singular locus of $\mathcal{A}_{2}$ has $H_{1}$ and $H_{4}$ as its two connected components. As analytic spaces, the surfaces $H_{1}$ and $H_{4}$ are each isomorphic to the Hilbert modular surface

$$
\left(\left(\mathrm{SL}_{2}(\mathbb{Z}) \times \mathrm{SL}_{2}(\mathbb{Z})\right) \rtimes \mathbb{Z}_{2}\right) \backslash(\mathbb{H} \times \mathbb{H}) .
$$

For a more detailed introduction to Siegel modular form, Humbert surfaces, and the Satake compactification of the Siegel modular threefold we refer to Freitag's book [6].

\subsection{Siegel modular forms}

In general, we can define the Eisenstein series $\psi_{2 k}$ of degree $g$ and weight $2 k$ (where we assume $2 k>g+1$ for convergence) by setting

$$
\psi_{2 k}(\underline{\tau})=\sum_{(C, D)} \operatorname{det}(C \cdot \underline{\tau}+D)^{-2 k}
$$

where the sum runs over non-associated bottom rows $(C, D)$ of elements in $\operatorname{Sp}_{2 g}(\mathbb{Z})$ where nonassociated means with respect to the multiplication by $\mathrm{GL}_{g}(\mathbb{Z})$. In the following, we will always assume $g=2$ in the definition of $\psi_{2 k}$. Using Igusa's definition [11, Section 8, p. 195] we define a cusp form of weight 10 by

$$
\chi_{10}(\underline{\tau})=-\frac{43867}{2^{12} 3^{5} 5^{2} 7 \cdot 53}\left(\psi_{4}(\underline{\tau}) \psi_{6}(\underline{\tau})-\psi_{10}(\underline{\tau})\right) .
$$

Based on Igusa's definition [11, Section 8, p. 195] and the work in [16] we define a second cusp form $\chi_{12}$ of weight 12 by

$$
\chi_{12}(\underline{\tau})=\frac{131 \cdot 593}{2^{13} 3^{7} 5^{3} 7^{2} 337}\left(3^{2} 7^{2} \psi_{4}^{3}(\underline{\tau})+2 \cdot 5^{3} \psi_{6}^{2}(\underline{\tau})-691 \psi_{12}(\underline{\tau})\right) .
$$


Moreover, Igusa proved $[12,13]$ that the ring of Siegel modular forms is generated by $\psi_{4}, \psi_{6}, \chi_{10}$, $\chi_{12}$ and by one more cusp form $\chi_{35}$ of odd weight 35 whose square is the following polynomial $[12$, p. 849$]$ in the even generators

$$
\begin{aligned}
\chi_{35}^{2}= & \frac{1}{2^{12} 3^{9}} \chi_{10}\left(2^{24} 3^{15} \chi_{12}^{5}-2^{13} 3^{9} \psi_{4}^{3} \chi_{12}^{4}-2^{13} 3^{9} \psi_{6}^{2} \chi_{12}^{4}+3^{3} \psi_{4}^{6} \chi_{12}^{3}-2 \cdot 3^{3} \psi_{4}^{3} \psi_{6}^{2} \chi_{12}^{3}\right. \\
& -2^{14} 3^{8} \psi_{4}^{2} \psi_{6} \chi_{10} \chi_{12}^{3}-2^{23} 3^{12} 5^{2} \psi_{4} \chi_{10}^{2} \chi_{12}^{3}+3^{3} \psi_{6}^{4} \chi_{12}^{3}+2^{11} 3^{6} 37 \psi_{4}^{4} \chi_{10}^{2} \chi_{12}^{2} \\
& +2^{11} 3^{6} 5 \cdot 7 \psi_{4} \psi_{6}^{2} \chi_{10}^{2} \chi_{12}^{2}-2^{23} 3^{9} 5^{3} \psi_{6} \chi_{10}^{3} \chi_{12}^{2}-3^{2} \psi_{4}^{7} \chi_{10}^{2} \chi_{12}+2 \cdot 3^{2} \psi_{4}^{4} \psi_{6}^{2} \chi_{10}^{2} \chi_{12} \\
& +2^{11} 3^{5} 5 \cdot 19 \psi_{4}^{3} \psi_{6} \chi_{10}^{3} \chi_{12}+2^{20} 3^{8} 5^{3} 11 \psi_{4}^{2} \chi_{10}^{4} \chi_{12}-3^{2} \psi_{4} \psi_{6}^{4} \chi_{10}^{2} \chi_{12}+2^{11} 3^{5} 5^{2} \psi_{6}^{3} \chi_{10}^{3} \chi_{12} \\
& -2 \psi_{4}^{6} \psi_{6} \chi_{10}^{3}-2^{12} 3^{4} \psi_{4}^{5} \chi_{10}^{4}+2^{2} \psi_{4}^{3} \psi_{6}^{3} \chi_{10}^{3}+2^{12} 3^{4} 5^{2} \psi_{4}^{2} \psi_{6}^{2} \chi_{10}^{4}+2^{21} 3^{7} 5^{4} \psi_{4} \psi_{6} \chi_{10}^{5} \\
& \left.-2 \psi_{6}^{5} \chi_{10}^{3}+2^{32} 3^{9} 5^{5} \chi_{10}^{6}\right) .
\end{aligned}
$$

Hence, the expression $Q:=2{ }^{12} 3^{9} \chi_{35}^{2} / \chi_{10}$ is a polynomial of degree 60 in the even generators. Igusa also proved that each Siegel modular form (with trivial character) of odd weight is divisible by the form $\chi_{35}$. The following fact is known[8]:

Proposition 2.1. The vanishing divisor of $Q$ is the Humbert surface $H_{4}$, i.e., a period point $\underline{\tau}$ is equivalent to a point with $\tau_{1}=\tau_{2}$ if and only if $Q=0$. Accordingly, the vanishing divisor of $\chi_{35}$ is the formal sum $H_{1}+H_{4}$ of Humbert surfaces, that constitutes the singular locus of $\mathcal{A}_{2}$.

In accordance with Igusa [11, Theorem 3] we also introduce the following ratios of Siegel modular forms

$$
\mathbf{x}_{1}=\frac{\psi_{4} \chi_{10}^{2}}{\chi_{12}^{2}}, \quad \mathbf{x}_{2}=\frac{\psi_{6} \chi_{10}^{3}}{\chi_{12}^{3}}, \quad \mathbf{x}_{3}=\frac{\chi_{10}^{6}}{\chi_{12}^{5}}
$$

as well as

$$
\mathbf{y}_{1}=\frac{\mathbf{x}_{1}^{3}}{\mathbf{x}_{3}}=\frac{\psi_{4}^{3}}{\chi_{12}}, \quad \mathbf{y}_{2}=\frac{\mathbf{x}_{2}^{2}}{\mathbf{x}_{3}}=\frac{\psi_{6}^{2}}{\chi_{12}}, \quad \mathbf{y}_{3}=\frac{\mathbf{x}_{1}^{2} \mathbf{x}_{2}}{\mathbf{x}_{3}}=\frac{\psi_{4}^{2} \psi_{6} \chi_{10}}{\chi_{12}}
$$

where we have suppressed the dependence of each Siegel modular form on $\underline{\tau}$. These ratios have the following asymptotic expansion for $z \rightarrow 0$ [11, pp. 180-182] in terms of ordinary Eisenstein series $E_{4}$ and $E_{6}$ and the Dedekind $\eta$-function

$$
\begin{aligned}
& \mathbf{x}_{1}=E_{4}\left(\tau_{1}\right) E_{4}\left(\tau_{2}\right)(\pi z)^{4}+O\left(z^{5}\right), \\
& \mathbf{x}_{2}=E_{6}\left(\tau_{1}\right) E_{6}\left(\tau_{2}\right)(\pi z)^{6}+O\left(z^{7}\right), \\
& \mathbf{x}_{3}=\eta^{24}\left(\tau_{1}\right) \eta^{24}\left(\tau_{2}\right)(\pi z)^{12}+O\left(z^{13}\right),
\end{aligned}
$$

and

$$
\begin{aligned}
& \mathbf{y}_{1}=j\left(\tau_{1}\right) j\left(\tau_{2}\right)+O\left(z^{2}\right), \\
& \mathbf{y}_{2}=\left(1728-j\left(\tau_{1}\right)\right)\left(1728-j\left(\tau_{2}\right)\right)+O\left(z^{2}\right), \\
& \mathbf{y}_{3}=\frac{E_{4}^{2}\left(\tau_{1}\right) E_{4}^{2}\left(\tau_{2}\right) E_{6}\left(\tau_{1}\right) E_{6}\left(\tau_{2}\right)}{\eta^{24}\left(\tau_{1}\right) \eta^{24}\left(\tau_{2}\right)}(\pi z)^{2}+O\left(z^{3}\right),
\end{aligned}
$$

where we have set

$$
\begin{aligned}
& j\left(\tau_{j}\right)=\frac{1728 E_{4}^{3}\left(\tau_{j}\right)}{E_{4}^{3}\left(\tau_{j}\right)-E_{6}^{2}\left(\tau_{j}\right)}=\frac{E_{4}^{3}\left(\tau_{j}\right)}{\eta^{24}\left(\tau_{j}\right)}, \\
& 1728-j\left(\tau_{j}\right)=\frac{1728 E_{6}^{2}\left(\tau_{j}\right)}{E_{4}^{3}\left(\tau_{j}\right)-E_{6}^{2}\left(\tau_{j}\right)}=\frac{E_{6}^{2}\left(\tau_{j}\right)}{\eta^{24}\left(\tau_{j}\right)} .
\end{aligned}
$$

The following fact follows from the above asymptotic analysis [8]:

Proposition 2.2. The modulus point $\underline{\tau}$ is equivalent to a point with $z=0$ or $[\underline{\tau}] \in H_{1} \subset \mathcal{A}_{2}$ such that the principally polarized abelian surface is a product of two elliptic curves $\mathbf{A}_{\underline{\tau}}=E_{\tau_{1}} \times E_{\tau_{2}}$ if and only if $\chi_{10}(\underline{\tau})=0$. The elliptic modular parameters are determined by equation (2.2). 


\subsection{Igusa invariants}

Suppose that $\mathcal{C}$ is an irreducible projective non-singular curve. If the self-intersection is $\mathcal{C} \cdot \mathcal{C}=2$ then $\mathcal{C}$ is a curve of genus two. For every curve $\mathcal{C}$ of genus two there exists a unique pair $\left(\operatorname{Jac}(\mathcal{C}), j_{\mathcal{C}}\right)$ where $\operatorname{Jac}(\mathcal{C})$ is an abelian surface, called the Jacobian variety of the curve $\mathcal{C}$, and $j_{\mathcal{C}}: \mathcal{C} \rightarrow \operatorname{Jac}(\mathcal{C})$ is an embedding. One can always regain $\mathcal{C}$ from the pair $(\operatorname{Jac}(\mathcal{C}), \mathcal{P})$ where $\mathcal{P}=[\mathcal{C}]$ is the class of $\mathcal{C}$ in the Néron-Severi group $\operatorname{NS}(\operatorname{Jac}(\mathcal{C}))$. Thus, if $\mathcal{C}$ is a genus-two curve, then $\operatorname{Jac}(\mathcal{C})$ is a principally polarized abelian surface with principal polarization $\mathcal{P}=[\mathcal{C}]$, and the map sending a curve $\mathcal{C}$ to its Jacobian variety $\operatorname{Jac}(\mathcal{C})$ is injective. In this way, the variety of moduli of curves of genus two is also the moduli space of their Jacobian varieties with canonical polarization.

We write the equation defining a genus-two curve $\mathcal{C}$ by a degree-six polynomial or sextic in the form

$$
\mathcal{C}: y^{2}=f(x)=a_{0} \prod_{i=1}^{6}\left(x-\alpha_{i}\right)=\sum_{i=0}^{6} a_{i} x^{i} .
$$

The roots $\left\{\alpha_{i}\right\}_{i=1}^{6}$ of the sextic are the six ramification points of the map $\mathcal{C} \rightarrow \mathbb{P}^{1}$. Their preimages on $\mathcal{C}$ are the six Weierstrass points. The isomorphism class of $f$ consists of all equivalent sextics where two sextics are considered equivalent if there is a linear transformation in $\mathrm{GL}_{2}(\mathbb{C})$ which takes the set of roots to the roots of the other.

The ring of invariants of binary sextics is generated by the Igusa invariants $\left(J_{2}, J_{4}, J_{6}, J_{10}\right)$ as defined in [14, equation (9)], which are the same invariants as the ones denoted by $\left(A^{\prime}, B^{\prime}, C^{\prime}, D^{\prime}\right)$ in $[18$, p. 319] and also the same invariants as $(A, B, C, D)$ in $[11$, p. 176]. For expressions of such invariants in terms of the coefficients $a_{0}, \ldots, a_{6}$ of the binary sextic, or $J_{k} \in \mathbb{Z}\left[a_{0}, \ldots, a_{6}\right]$ for $k \in\{2,4,6,10\}$; see [14, equation (11)] and in terms of thetanulls see [17]. One can then ask what the Igusa invariants of a genus-two curve $\mathcal{C}$ defined by a sextic curve $f$ are in terms of $\underline{\tau}$ such that $\left(\underline{\tau}, \mathbb{I}_{2}\right) \in \operatorname{Mat}(2,4 ; \mathbb{C})$ is the period matrix of the principally polarized abelian surface $\mathbf{A}_{\underline{\tau}}=\operatorname{Jac}(\mathcal{C})$. Based on the asymptotic behavior in Equations (2.2) and (2.2), Igusa [12, p. 848] proved that the relations are as follows

$$
\begin{aligned}
& J_{2}=-2^{3} \cdot 3 \frac{\chi_{12}(\underline{\tau})}{\chi_{10}(\underline{\tau})}, \\
& J_{4}=2^{2} \psi_{4}(\underline{\tau}), \\
& J_{6}=-\frac{2^{3}}{3} \psi_{6}(\underline{\tau})-2^{5} \frac{\psi_{4}(\underline{\tau}) \chi_{12}(\underline{\tau})}{\chi_{10}(\underline{\tau})}, \\
& J_{10}=-2^{14} \chi_{10}(\underline{\tau}) .
\end{aligned}
$$

Thus, the invariants of a sextic define a point in a weighted projective space $\left[J_{2}: J_{4}: J_{6}\right.$ : $\left.J_{10}\right] \in \mathbb{W P}_{(2,4,6,10)}^{3}$ that equals

$$
\left[2^{3} 3\left(3 \chi_{12}\right): 2^{2} 3^{2} \psi_{4} \chi_{10}^{2}: 2^{3} 3^{2}\left(4 \psi_{4}\left(3 \chi_{12}\right)+\psi_{6} \chi_{10}\right) \chi_{10}^{2}: 2^{2} \chi_{10}^{6}\right] .
$$

Torelli's theorem states that the map sending a curve $\mathcal{C}$ to its Jacobian variety $\operatorname{Jac}(\mathcal{C})$ induces a birational map from the moduli space $\mathcal{M}_{2}$ of genus-two curves to the complement of the Humbert surface $H_{1}$ in $\mathcal{A}_{2}$, i.e., $\mathcal{A}_{2}-\operatorname{supp}\left(\chi_{10}\right)_{0}$. In other words, points in the projective variety $\operatorname{Proj} \mathbb{C}\left[J_{2}, J_{4}, J_{6}, J_{10}\right]$ which are not on $J_{10}=0$ are in one-to-one correspondence with isomorphism classes of regular sextics [11].

Often the Clebsch invariants $(A, B, C, D)$ of a sextic are used instead. They are defined in terms of the transvectants of the binary sextics; see [5] for details. The invariants $(A, B, C, D)$ are polynomial expressions in the Igusa invariants $\left(J_{2}, J_{4}, J_{6}, J_{10}\right)$ with rational coefficients:

$$
A=-\frac{1}{2^{3} 3 \cdot 5} J_{2}
$$




$$
\begin{aligned}
& B=\frac{1}{2^{3} 3^{3} 5^{4}}\left(J_{2}^{2}+20 J_{4}\right), \\
& C=-\frac{1}{2^{5} 3^{5} 5^{6}}\left(J_{2}^{3}+80 J_{2} J_{4}-600 J_{6}\right), \\
& D=-\frac{1}{2^{8} 3^{9} 5^{10}}\left(9 J_{2}^{5}+700 J_{2}^{3} J_{4}-3600 J_{2}^{2} J_{6}-12400 J_{2} J_{4}^{2}+48000 J_{4} J_{6}+10800000 J_{10}\right) .
\end{aligned}
$$

For formulas giving relations between all these sets of invariants see [1].

\subsubsection{Absolute invariants}

Dividing any $\mathrm{SL}_{2}(\mathbb{C})$ invariant by another one of the same degree gives an invariant under $\mathrm{GL}_{2}(\mathbb{C})$ action. The term absolute invariants is used first by Igusa [10] for $\mathrm{GL}_{2}(\mathbb{C})$ invariants. It was the main result of $[11$, Theorem 3$]$ that

$$
\mathbf{x}_{1}=144 \frac{J_{4}}{J_{2}^{2}}, \quad \mathbf{x}_{2}=-1728 \frac{J_{2} J_{4}-3 J_{6}}{J_{2}^{3}}, \quad \mathbf{x}_{3}=486 \frac{J_{10}}{J_{2}^{5}},
$$

for $J_{2} \neq 0$. We use $\mathbf{x}_{1}, \mathbf{x}_{2}, \mathbf{x}_{3}$ to write the point $\left[J_{2}: J_{4}: J_{6}: J_{10}\right] \in \mathbb{W P}_{(2,4,6,10)}^{3}$ as

$$
\left[1: \frac{1}{2^{4} 3^{2}} \mathbf{x}_{1}: \frac{1}{2^{6} 3^{4}} \mathbf{x}_{2}+\frac{1}{2^{4} 3^{3}} \mathbf{x}_{1}: \frac{1}{2 \cdot 3^{5}} \mathbf{x}_{3}\right]
$$

Since the invariants $J_{4}, J_{6}, J_{10}$ vanish simultaneously for sextics with triple roots all such curves are mapped to $[1: 0: 0: 0] \in \mathbb{W P}_{(2,4,6,10)}^{3}$ with uniformizing affine coordinates $\mathbf{x}_{1}, \mathbf{x}_{2}, \mathbf{x}_{3}$ around it. Blowing up this point gives a variety that parameterizes genus-two curves with $J_{2} \neq 0$ and their degenerations. In the blow-up space we have to introduce additional coordinates that are obtained as ratios of $\mathbf{x}_{1}, \mathbf{x}_{2}, \mathbf{x}_{3}$ and have weight zero. Those are precisely the coordinates $\mathbf{y}_{1}, \mathbf{y}_{2}, \mathbf{y}_{3}$ already introduced in equation (2.1). It turns out that the coordinate ring of the blown-up space is $\mathbb{C}\left[\mathbf{x}_{1}, \mathbf{x}_{2}, \mathbf{x}_{3}, \mathbf{y}_{1}, \mathbf{y}_{2}, \mathbf{y}_{3}\right]$.

We introduce the three absolute invariants

$$
\begin{aligned}
\rho= & -\frac{4\left(9 J_{2}^{2}-320 J_{4}\right)\left(J_{2}^{2}+20 J_{4}\right)^{2}}{\left(3 J_{2}^{3}+140 J_{2} J_{4}-800 J_{6}\right)^{2}}, \\
\sigma= & -\frac{48\left(J_{2}^{2}+20 J_{4}\right)^{2}}{\left(3 J_{2}^{3}+140 J_{2} J_{4}-800 J_{6}\right)^{3}} \\
& \times\left(9 J_{2}^{5}-700 J_{2}^{3} J_{4}+2400 J_{2}^{2} J_{6}-262400 J_{2} J_{4}^{2}+768000 J_{4} J_{6}+172800000 J_{10}\right), \\
\kappa= & \frac{2\left(27 J_{2}^{4}+2380 J_{2}^{2} J_{4}-12000 J_{2} J_{6}+12800 J_{4}^{2}\right)\left(J_{2}^{2}+20 J_{4}\right)}{\left(3 J_{2}^{3}+140 J_{2} J_{4}-800 J_{6}\right)^{2}} .
\end{aligned}
$$

It follows:

Lemma 2.3. For invariants $(\rho, \sigma, \kappa)$ given by equation (2.5) such that $\rho$ and $\kappa$ do not vanish simultaneously, a point $\left[J_{2}: J_{4}: J_{6}: J_{10}\right]$ in $\mathbb{W P}_{(2,4,6,10)}^{3}$ is given by

$$
\begin{aligned}
J_{2}= & 8(\kappa-\rho), \quad J_{4}=\frac{9}{5}(\kappa-\rho)^{2}+45 \rho, \\
J_{6}= & \frac{111}{25}(\kappa-\rho)^{3}-30(\kappa-\rho)^{2}+63 \rho(\kappa-\rho)-270 \rho, \\
J_{10}= & \frac{6}{3125}(\kappa-\rho)^{5}+\frac{4}{15}(\kappa-\rho)^{4}+\frac{46}{75} \rho(\kappa-\rho)^{3} \\
& +\left(-\frac{1}{6} \sigma+\frac{42}{5} \rho\right)(\kappa-\rho)^{2}+12 \rho^{2}(\kappa-\rho)+\frac{3}{2} \rho(36 \rho-\sigma) .
\end{aligned}
$$

In particular, for $J_{2} \neq 0$ we have $\mathbb{Q}\left(\mathbf{x}_{1}, \mathbf{x}_{2}, \mathbf{x}_{3}\right)=\mathbb{Q}(\rho, \sigma, \kappa)$. 
Proof. The proof is computational. We express $\rho, \sigma, \kappa$ as rational functions of $\mathbf{x}_{1}, \mathbf{x}_{2}, \mathbf{x}_{3}$ and vice versa over $\mathbb{Q}$. The condition that $\rho$ and $\kappa$ do not vanish simultaneously is based on the fact that $J_{2}, J_{4}, J_{6}, J_{10}$ must not vanish simultaneously.

Remark 2.4. Consider the image of $\left[J_{2}: J_{4}: J_{6}: J_{10}\right]$ in $\mathbb{W}_{(2,4,6,10)}^{3}$ under the morphism $\mathbb{W P}_{(2,4,6,10)}^{3} \rightarrow \mathbb{P}^{5}$ given by

$$
\left[486 J_{4} J_{6}: 486 J_{10}:-1728\left(J_{2} J_{4}-3 J_{6}\right) J_{2}^{2}: 144 J_{2}^{3} J_{4}: 20736 J_{2} J_{4}^{2}: J_{2}^{5}\right],
$$

which is a linear transformation of the usual morphism to $\mathbb{P}^{5}$ given by

$$
\left[J_{2}: J_{4}: J_{6}: J_{10}\right] \mapsto\left[J_{10}: J_{4} J_{6}: J_{2}^{2} J_{6}: J_{2}^{3} J_{4}: J_{2} J_{4}^{2}: J_{2}^{5}\right] .
$$

For $J_{2} \neq 0$, points in equation $(2.7)$ equal

$$
\left[\frac{1}{1536} \mathbf{x}_{1}\left(\mathbf{x}_{2}+12 \mathbf{x}_{1}\right): \mathbf{x}_{3}: \mathbf{x}_{2}: \mathbf{x}_{1}: \mathbf{x}_{1}^{2}: 1\right]
$$

The invariants $\mathbf{x}_{1}, \mathbf{x}_{2}, \mathbf{x}_{3}$ are not defined for $J_{2}=0$, but $\rho, \sigma, \kappa$ remain well-defined if $\rho=\kappa \neq 0$. In this case we have

$$
J_{2}^{(0)}=0, \quad J_{4}^{(0)}=45 \rho, \quad J_{6}^{(0)}=-270 \rho, \quad J_{10}^{(0)}=\frac{3}{2} \rho(36 \rho-\sigma),
$$

and the invariants $\rho$ and $\sigma$ with

$$
\rho=\kappa=\frac{4}{5} \frac{J_{4}^{3}}{J_{6}^{2}}, \quad \sigma=\frac{144}{5} \frac{J_{4}^{3}}{J_{6}^{2}}+6480 \frac{J_{4}^{3}}{J_{6}^{2}} \frac{J_{10}}{J_{4} J_{6}},
$$

determine genus-two curves with $J_{2}=0, J_{4} \cdot J_{6} \neq 0$ up to isomorphism. In addition to $J_{2}=0$, we have $J_{10}=0$ if and only if $\sigma=36 \rho$. Using $\epsilon=(\kappa-\rho)$ in equation (2.6), one checks that points in equation (2.7) up to terms of order $O\left(\epsilon^{2}\right)$ equal

$$
\left[1-\frac{7}{30} \epsilon: \frac{J_{10}^{(0)}}{J_{4}^{(0)} J_{6}^{(0)}}-\frac{2}{2025} \epsilon: 0: 0:-\frac{512}{9} \epsilon: 0\right] .
$$

This means that under the usual morphism to $\mathbb{P}^{5}$ the regular genus-two curves with $J_{2}=0$ and constant ratio $J_{10} /\left(J_{4} J_{6}\right)$ are mapped to the same point.

\subsection{Recovering the equation of the curve from invariants}

Let $\mathfrak{p} \in \mathcal{M}_{2}$ and $\mathcal{C}$ a genus-two curve corresponding to $\mathfrak{p}$ defined by the sextic polynomial $f$ in equation (2.3). Then, Aut( $\mathfrak{p})$ is a finite group as described in [19]. The quotient space $\mathcal{C} / \operatorname{Aut}(\mathfrak{p})$ is a genus zero curve and therefore isomorphic to a conic. Since conics are in one to one correspondence with three-by-three symmetric matrices (up to equivalence), let $M=\left[A_{i j}\right]$ be the symmetric matrix corresponding to this conic. Let $\mathbf{X}=\left[X_{1}: X_{2}: X_{3}\right] \in \mathbb{P}^{2}$ and

$$
\mathcal{Q}: \mathbf{X}^{t} \cdot M \cdot \mathbf{X}=\sum_{i, j=1}^{3} A_{i j} X_{i} X_{j}=0
$$

Clebsch [5] determined the entries of this matrix $M$ as follows

$$
A_{11}=2 C+\frac{1}{3} A B
$$




$$
\begin{aligned}
& A_{22}=A_{13}=D, \\
& A_{33}=\frac{1}{2} B D+\frac{2}{9} C\left(B^{2}+A C\right), \\
& A_{23}=\frac{1}{3} B\left(B^{2}+A C\right)+\frac{1}{3} C\left(2 C+\frac{1}{3} A B\right), \\
& A_{12}=\frac{2}{3}\left(B^{2}+A C\right) .
\end{aligned}
$$

The coefficients are obtained as follows: from the sextic $f$ in equation (2.3) three binary quadrics $\mathrm{y}_{i}(x)$ with $i=1,2,3$ are obtained by an operation called 'Überschiebung' [18, p. 317] or transvection. The quadrics $\mathrm{y}_{i}$ for $i=1,2,3$ have the property that their coefficients are polynomial expressions in the coefficients of $f$ with rational coefficients. Moreover, under the operation $f(x) \mapsto \tilde{f}(x)=f(-x)$ the quadrics change according to $\mathrm{y}_{i}(x) \mapsto \tilde{\mathrm{y}}_{i}(x)=\mathrm{y}_{i}(-x)$ for $i=1,2,3$. Hence, they are not invariants of the sextic $f$. The coefficients $A_{i j}$ in equation (2.9) satisfy $A_{i j}=\left(\mathrm{y}_{i} \mathrm{y}_{j}\right)_{2} \cdot{ }^{1}$ Therefore, the coefficients $A_{i j}$ are invariant under the operation $f(x) \mapsto$ $\tilde{f}(x)=f(-x)$, and the locus $D=0$ is equivalent to

$$
D=0 \quad \Leftrightarrow \quad\left(\mathrm{y}_{1} \mathrm{y}_{3}\right)_{2}=\left(\mathrm{y}_{2} \mathrm{y}_{2}\right)_{2}=0 .
$$

We define $R$ to be $1 / 2$ times the determinant of the three binary quadrics $\mathrm{y}_{i}$ for $i=1,2,3$ with respect to the basis $x^{2}, x, 1$. If one extends the operation of Überschiebung by product rule [18, p. 317], then $R$ can be re-written as

$$
R=-\left(\mathrm{y}_{1} \mathrm{y}_{2}\right)_{1}\left(\mathrm{y}_{2} \mathrm{y}_{3}\right)_{1}\left(\mathrm{y}_{3} \mathrm{y}_{1}\right)_{1}
$$

or, equivalently, as

$$
\begin{aligned}
R= & -\frac{1}{8}\left(\mathrm{y}_{1, y y} \mathrm{y}_{2, x y} \mathrm{y}_{3, x x}-\mathrm{y}_{1, y y} \mathrm{y}_{2, x x} \mathrm{y}_{3, x y}-\mathrm{y}_{1, x y} \mathrm{y}_{2, y y} \mathrm{y}_{3, x x}\right. \\
& \left.+\mathrm{y}_{1, x y} \mathrm{y}_{2, x x} \mathrm{y}_{3, y y}+\mathrm{y}_{1, x x} \mathrm{y}_{2, y y} \mathrm{y}_{3, x y}-\mathrm{y}_{1, x x} \mathrm{y}_{2, x y} \mathrm{y}_{3, y y}\right) .
\end{aligned}
$$

It is then obvious that under the operation $f(x) \mapsto \tilde{f}(x)=f(-x)$ the determinant $R$ changes its sign, i.e., $R(f) \mapsto R(\tilde{f})=-R(f)$. A straightforward calculation shows that

$$
R^{2}=\frac{1}{2}\left|\begin{array}{lll}
A_{11} & A_{12} & A_{13} \\
A_{12} & A_{22} & A_{23} \\
A_{13} & A_{23} & A_{33}
\end{array}\right|,
$$

where $A_{i j}$ are the invariants in equation (2.9). Like the coefficients $A_{i j}, R^{2}$ is invariant under the operation $f(x) \mapsto \tilde{f}(x)=f(-x)$ and must be a polynomial in $\left(J_{2}, J_{4}, J_{6}, J_{10}\right)$. Substituting $(2.3)$ into the Clebsch invariants and then equation (2.9) it follows that

$$
R^{2}=\left(2^{9} 3^{-9} 5^{-10} i \frac{\chi_{35}(\underline{\tau})}{\chi_{10}(\underline{\tau})^{2}}\right)^{2} .
$$

Bolza [2] described the possible automorphism groups of genus-two curves defined by sextics and provided criteria for the cases when the automorphism group of the sextic curve in equation (2.3) is nontrivial. For a detailed discussion of the automorphism groups of genus-two curve defined over any field $k$ and the corresponding loci in $\mathcal{M}_{2}$ see [19]. We have the following lemma summarizing our discussion:

\footnotetext{
${ }^{1}$ For two binary forms $f, g$ of degree $m$ and $n$, respectively, we denote the Überschiebung of order $k$ by $(f g)_{k}=(-1)^{k}(g f)_{k}$. For $\tilde{f}(x)=f(-x)$ and $\tilde{g}(x)=g(-x)$ and $m=n=k$, we have $(f g)_{m}=(-1)^{m}(\tilde{f} \tilde{g})_{m}$.
} 
Lemma 2.5. We have the following statements:

1. $R^{2}$ is an order 30 invariant of binary sextics expressed as a polynomial in $\left(J_{2}, J_{4}, J_{6}, J_{10}\right)$ as in [19, equation (17)] given by plugging Clebsch invariants and (2.9) into equation (2.10).

2. The locus of curves $\mathfrak{p} \in \mathcal{M}_{2}$ such that $V_{4} \hookrightarrow \operatorname{Aut}(\mathfrak{p})$ is a two-dimensional irreducible rational subvariety of $\mathcal{M}_{2}$ given by the equation $R^{2}=0$ and a birational parametrization given by the $u, v$-invariants as in [19, Theorem 1].

We have introduced the invariant $R^{2}$ for any binary sextic $f$. To the corresponding symmetric matrix $M$ with coefficients $A_{i j}=\left(\mathrm{y}_{i} \mathrm{y}_{j}\right)_{2}$ of order zero and invariant under the operation $f(x) \mapsto$ $\tilde{f}(x)=f(-x)$, we associated a conic $\mathcal{Q}$. Similarly, there is also a cubic curve given by the equation

$$
\mathcal{T}: \sum_{1 \leq i, j, k \leq 3} a_{i j k} X_{i} X_{j} X_{k}=0
$$

where the coefficients $a_{i j k}$ are of order zero and invariant under $f(x) \mapsto \tilde{f}(x)=f(-x)$. In terms of 'Überschiebung' the coefficients are obtained by

$$
a_{i j k}=\left(f \mathrm{y}_{i}\right)_{2}\left(f \mathrm{y}_{j}\right)_{2}\left(f \mathrm{y}_{k}\right)_{2} .
$$

The coefficients $a_{i j k}$ are given explicitly as follows:

$$
\begin{aligned}
& 36 a_{111}=8\left(A^{2} C-6 B C+9 D\right), \\
& 36 a_{112}=4\left(2 B^{3}+4 A B C+12 C^{2}+3 A D\right), \\
& 36 a_{113}=36 a_{122}=4\left(A B^{3}+4 / 3 A^{2} B C+4 B^{2} C+6 A C^{2}+3 B D\right), \\
& 36 a_{123}=2\left(2 B^{4}+4 A B^{2} C+4 / 3 A^{2} C^{2}+4 B C^{2}+3 A B D+12 C D\right), \\
& 36 a_{133}=2\left(A B^{4}+4 / 3 A^{2} B^{2} C+16 / 3 B^{3} C+26 / 3 A B C^{2}+8 C^{3}+3 B^{2} D+2 A C D\right), \\
& 36 a_{222}=4\left(3 B^{4}+6 A B^{2} C+8 / 3 A^{2} C^{2}+2 B C^{2}-3 C D\right), \\
& 36 a_{223}=2\left(-2 / 3 B^{3} C-4 / 3 A B C^{2}-4 C^{3}+9 B^{2} D+8 A C D\right), \\
& 36 a_{233}=2\left(B^{5}+2 A B^{3} C+8 / 9 A^{2} B C^{2}+2 / 3 B^{2} C^{2}-B C D+9 D^{2}\right), \\
& 36 a_{333}=-2 B^{4} C-4 A B^{2} C^{2}-16 / 9 A^{2} C^{3}-4 / 3 B C^{3}+9 B^{3} D+12 A B C D+20 C^{2} D .
\end{aligned}
$$

The relations between all aforementioned invariants and Siegel modular forms, in particular the relation between $\chi_{35}$ and $R^{2}$ can be found in [1].

Since 'Überschiebung' preserves the rationality of the coefficients, we have the following corollary:

Corollary 2.6. Let $\mathfrak{p} \in \mathcal{M}_{2}$ and $\mathcal{C}$ a genus-two curve corresponding to $\mathfrak{p}$ defined by a sextic polynomial $f$ in equation (2.3). Then, Aut $(\mathfrak{p})$ is a finite group, and the quotient space $\mathcal{C} /$ Aut $(\mathfrak{p})$ is a genus zero curve isomorphic to the conic $\mathcal{Q}$ in equation (2.8). Moreover, if $\mathfrak{p} \in \mathcal{M}_{2}(K)$, for some number field $K$, the conic $\mathcal{Q}$ and cubic $\mathcal{T}$ have $K$-rational coefficients.

The intersection of the conic $\mathcal{Q}$ with the cubic $\mathcal{T}$ consists of six points which are the zeroes of a polynomial $f(x)$ of degree 6 in the parameter $x$. The roots of this polynomial are the images of the Weierstrass points under the hyperelliptic projection. Hence, the affine equation of a genus-two curve corresponding to $\mathfrak{p}$ is given by $y^{2}=f(x)$. The main question is if the sextic given by $y^{2}=f(x)$ provides a genus-two curve defined over a minimal field of definition. We start with the following known result.

Proposition 2.7. A genus $g \geq 2$ hyperelliptic curve $\mathcal{X}_{g}$ with hyperelliptic involution $w$ is defined over the $K$ if and only if the conic $\mathcal{Q}=\mathcal{X}_{g} /\langle w\rangle$ has a $K$-rational point. 
The above result was briefly described in [18, Lemma 1] even though it seems as it had been known before. Mestre's method is briefly described as follows: if the conic $\mathcal{Q}$ has a rational point over $\mathbb{Q}$, then this leads to a parametrization of $\mathcal{Q}$, say $\left(h_{1}(x), h_{2}(x), h_{3}(x)\right)$. Substitute $X_{1}, X_{2}, X_{3}$ by $h_{1}(x), h_{2}(x), h_{3}(x)$ in the cubic $\mathcal{T}$ and we get the degree 6 polynomial $f(x)$. However, if the conic has no rational point or $R^{2}=\frac{1}{2} \operatorname{det} M=0$ the method obviously fails. In Section 3 we determine the intersection $\mathcal{T} \cap \mathcal{Q}$ over a quadratic extension which is always possible.

\section{A universal genus-two curve from the moduli space}

The goal of this section is to explicitly determine a universal equation of a genus-two curve corresponding to this generic point $\mathfrak{p}$. We have the following lemma:

Lemma 3.1. The conic $\mathcal{Q}$ in equation $(2.8)$ for $J_{4} \cdot J_{6} \cdot J_{10} \neq 0$ is equivalent over $\mathbb{Q}\left[J_{2}, \rho, \sigma, \kappa\right]$ to the conic

$$
\mathcal{Q}^{\prime}: x_{1}^{2}-\gamma x_{2}^{2}-\Lambda_{6} x_{3}^{2}=0,
$$

where $(\rho, \sigma, \kappa)$ are the absolute invariants in equation $(2.5), \gamma=\rho^{2}+\sigma$ and

$$
\begin{aligned}
\Lambda_{6}= & -\gamma^{3}-27 \rho \gamma^{2}-81 \rho^{2}(\rho+12) \gamma+729 \rho^{2}(\rho+12)^{2} \\
& +\left(-6 \rho \gamma^{2}+54 \rho(5 \rho+36) \gamma-1944 \rho^{2}(\rho+12)\right) \kappa \\
& +\left(9 \gamma^{2}-9 \rho(\rho+36) \gamma+162 \rho\left(\rho^{2}+32 \rho+144\right)\right) \kappa^{2} \\
& +((30 \rho+216) \gamma-432 \rho(\rho+12)) \kappa^{3}+\left(9 \rho^{2}-24 \gamma+504 \rho+1296\right) \kappa^{4} \\
& +(-24 \rho-288) \kappa^{5}+16 \kappa^{6} .
\end{aligned}
$$

Moreover, for $J_{2}, \rho, \sigma, \kappa \in \mathbb{Q}$ the conic $\mathcal{Q}$ in equation (2.8) has a rational point if and only if the conic $\mathcal{Q}^{\prime}$ in equation (3.1) does.

Proof. For the conic $\mathcal{Q}$ in equation (2.8), we apply the coordinate transformation given by

$$
\begin{aligned}
X_{1}= & 2(A B+6 C)^{4}\left(A C+B^{2}\right) x_{1} \\
& +108 B(A B+6 C)^{2}\left(4 A^{2} C^{2}+8 A B^{2} C+4 B^{4}-3 A B D-18 C D\right) x_{2} \\
& +41990 B^{3}\left(8 A^{2} B C^{2}+14 A B^{3} C+6 B^{5}+12 A C^{3}+12 B^{2} C^{2}-27 D^{2}\right) x_{3}, \\
X_{2}= & -(A B+6 C)^{5} x_{1}-419904 B^{3} x_{3} \\
& \times\left(4 A^{2} B^{2} C+3 A B^{4}+30 A B C^{2}+18 B^{3} C-18 A C D-18 B^{2} D+36 C^{3}\right), \\
X_{3}= & -2^{6} 3^{9} B^{3}\left(4 A^{2} C^{2}+8 A B^{2} C+4 B^{4}-3 A B D-18 C D\right) x_{3} .
\end{aligned}
$$

We then obtain the conic $\mathcal{Q}^{\prime}$ in equation (3.1). Equation (3.3) can be rewritten as transformation over $\mathbb{Q}\left[J_{2}, \rho, \sigma, \kappa\right]$ using equations $(2.4)$ and $(2.5)$.

We have the following lemma:

Lemma 3.2. Assume $\rho, \sigma, \kappa \in \mathbb{Q}$. The conic $\mathcal{Q}^{\prime}$ in equation (3.1) has a rational point if and only if there are rational numbers $\alpha, \beta \in \mathbb{Q}$ such that

$$
\alpha^{2}+\Lambda_{6} \beta^{2} \sigma=\gamma .
$$

The rational point on the conic $\mathcal{Q}^{\prime}$ is then given by

$$
\left[x_{1}^{0}: x_{2}^{0}: x_{3}^{0}\right]=[\alpha \rho+\gamma: \alpha+\rho: \beta \sigma] .
$$

Conversely, every rational point on the conic $\mathcal{Q}^{\prime}$ can be written in the form of equation (3.5) for some rational numbers $\alpha, \beta \in \mathbb{Q}$ satisfying equation (3.4). 
Proof. If rational numbers $\alpha, \beta$ exist such that equation (3.4) is satisfied, then the point in equation (3.5) is rational and is easily checked to be on the conic. If there is a rational point on the conic then we can choose $\beta \in \mathbb{Q}$ in equation (3.5), thus $\alpha \in \mathbb{Q}$.

We have the following:

Lemma 3.3. Assume that a point on the conic in equation (3.1) is given by equation (3.5) with $x_{2}^{0} \neq 0$ which is always possible if $\rho \neq 0$. Then every point on the conic is given by

$$
\begin{aligned}
& x_{1}=(\alpha \rho+\gamma) U^{2}+2 \Lambda_{6} \beta \sigma U V+\Lambda_{6}(\alpha \rho+\gamma) V^{2}, \\
& x_{2}=(\alpha+\rho) U^{2}-\Lambda_{6}(\alpha+\rho) V^{2}, \\
& x_{3}=\beta \sigma U^{2}+2(\alpha \rho+\gamma) U V+\Lambda_{6} \beta \sigma V^{2},
\end{aligned}
$$

for some $[U: V] \in \mathbb{P}^{1}$. The parametrization in equation (3.6) is a rational parametrization of the conic $\mathcal{Q}^{\prime}$ if and only if $\alpha, \beta, \rho, \kappa, \sigma \in \mathbb{Q}$.

Proof. If a point of $\mathcal{Q}^{\prime}$ is obtained from some (rational) values $(\alpha, \beta)$ then there are three more (rational) points given by setting $(\alpha, \beta) \mapsto( \pm \alpha, \pm \beta)$. If $\rho \neq 0$, one of these points satisfies $x_{2}^{0}=\alpha+\rho \neq 0$. The proof then follows from the known formulas parametrizing conics for $x_{2}^{0} \neq 0$ given by

$$
\begin{aligned}
& x_{1}=a x_{1}^{0} U^{2}-2 c x_{3}^{0} U V-c x_{1}^{0} V^{2}, \\
& x_{2}=a x_{2}^{0} U^{2}+c x_{2}^{0} V^{2}, \\
& x_{3}=a x_{3}^{0} U^{2}+2 a x_{1}^{0} U V-c x_{3}^{0} V^{2},
\end{aligned}
$$

where $a=1, b=-\gamma, c=-\Lambda_{6}$ and $x_{1}^{0}, x_{2}^{0}, x_{3}^{0}$ were given in equation (3.5).

Remark 3.4. If $\alpha=\rho=0$ and $\gamma \neq 0$, a formula similar to equation (3.6) can be found using the fact that $x_{1}^{0} \neq 0$ in equation (3.5) in this case.

Remark 3.5. If a point of $\mathcal{Q}^{\prime}$ is obtained for some (rational) values $(\alpha, \beta)$ then three more (rational) points on $\mathcal{Q}^{\prime}$ are given by setting $(\alpha, \beta) \mapsto( \pm \alpha, \pm \beta)$ in equation (3.5).

Changing from coordinates $\left[X_{1}: X_{2}: X_{3}\right]$ to coordinates $\left[x_{1}: x_{2}: x_{3}\right]$ transforms the conic $\mathcal{Q}$ in equation (2.8) into the conic $\mathcal{Q}^{\prime}$ in equation (3.1). Similarly, under the same change of coordinates the cubic $\mathcal{T}$ in equation (2.11) becomes

$$
\begin{aligned}
\Lambda_{1}(18 \gamma+ & \left.\Lambda_{3}\right) x_{1}^{3}+\gamma^{3} \Lambda_{2} x_{2}^{3}-\left(\gamma-\Lambda_{1}\right) \Lambda_{6}^{2} x_{3}^{3}+3 \Lambda_{1} \Lambda_{6} x_{1}^{2} x_{3}+3 \gamma\left(9 \gamma \rho+\kappa \Lambda_{3}\right) x_{1}^{2} x_{2} \\
& +3 \gamma^{2} \Lambda_{3} x_{1} x_{2}^{2}+3 \Lambda_{5} \Lambda_{6} x_{1} x_{3}^{2}+3 \gamma \Lambda_{4} \Lambda_{6} x_{2} x_{3}^{2}+3 \gamma^{2} \Lambda_{6} x_{2}^{2} x_{3}+6 \gamma \kappa \Lambda_{6} x_{1} x_{2} x_{3},
\end{aligned}
$$

with coefficients given by

$$
\begin{aligned}
\Lambda_{1}= & 9 \rho+\kappa^{2}, \\
\Lambda_{2}= & \gamma+18 \rho+3 \rho \kappa-4 \kappa^{2}, \\
\Lambda_{3}= & 27 \rho(\rho+12)+(\gamma-36 \rho) \kappa+3(\rho+12) \kappa^{2}-4 \kappa^{3}, \\
\Lambda_{4}= & -\gamma^{2}-9 \gamma \rho-3(\gamma \rho-9 \rho(\rho+12)) \kappa+(5 \gamma-36 \rho) \kappa^{2}+3(\rho+12) \kappa^{3}-4 \kappa^{4}, \\
\Lambda_{5}= & -27 \gamma \rho(\rho+6)+243 \rho^{2}(\rho+12)-\left(\gamma^{2}-45 \gamma \rho+324 \rho^{2}\right) \kappa \\
& -(3 \gamma(\rho-6)-54 \rho(\rho+12)) \kappa^{2}+(5 \gamma-72 \rho) \kappa^{3}+3(\rho+12) \kappa^{4}-4 \kappa^{5} .
\end{aligned}
$$

We also discuss the conic, cubic, rational point and parametrization in the cases where $J_{2}=0$ and $J_{4} \cdot J_{6}=0$ : 
Lemma 3.6. If $J_{2}=J_{4}=0$ and $J_{6} \cdot J_{10} \neq 0$, the conic $\mathcal{Q}$ in equation (2.8) is equivalent over $\mathbb{Q}\left[J_{6}, J_{10}\right]$ to the conic

$$
\mathcal{Q}^{\prime}: x_{1}^{2}-\mu x_{2}^{2}-(1-\mu) x_{3}^{2}=0,
$$

with $\mu=J_{6}^{5} /\left(2^{4} 3^{4} 5^{5} J_{10}^{3}\right)$ and a rational point given by $\left[x_{1}^{0}: x_{2}^{0}: x_{3}^{0}\right]=[1: 1: 1]$. A rational parametrization of $\mathcal{Q}^{\prime}$ is then given by

$$
\begin{aligned}
& x_{1}=U^{2}+2(1-\mu) U V+(1-\mu) V^{2}, \quad x_{2}=U^{2}-(1-\mu) V^{2}, \\
& x_{3}=U^{2}+2 U V+(1-\mu) V^{2}
\end{aligned}
$$

with $[U: V] \in \mathbb{P}^{1}$. Under the same change of coordinates the cubic $\mathcal{T}$ in equation (2.11) becomes

$$
\begin{aligned}
\mathcal{T}^{\prime}: 0= & 2 x_{1}^{3}-\mu^{2} x_{2}^{3}-2(1-\mu)^{2} x_{3}^{3}-6 \mu x_{1}^{2} x_{2} \\
& -6(1-\mu) x_{1}^{2} x_{3}+6(1-\mu) x_{1} x_{3}^{2}-3 \mu(1-\mu) x_{2} x_{3}^{2} .
\end{aligned}
$$

If $J_{2}=J_{6}=0$ and $J_{4} \cdot J_{10} \neq 0$, the conic $\mathcal{Q}$ in equation $(2.8)$ is equivalent over $\mathbb{Q}\left[J_{4}, J_{10}\right]$ to the conic

$$
\mathcal{Q}^{\prime}: x_{1}^{2}-x_{2}^{2}-(1-\nu) x_{3}^{2}=0,
$$

with $\nu=J_{4}^{5} /\left(2^{2} 3^{5} 5^{5} J_{10}^{2}\right)$ and a rational point given by $\left[x_{1}^{0}: x_{2}^{0}: x_{3}^{0}\right]=[1: 1: 0]$. A rational parametrization of $\mathcal{Q}^{\prime}$ is then given by

$$
x_{1}=U^{2}+(1-\nu) V^{2}, \quad x_{2}=U^{2}-(1-\nu) V^{2}, \quad x_{3}=2 U V
$$

with $[U: V] \in \mathbb{P}^{1}$. Under the same change of coordinates the cubic $\mathcal{T}$ in equation (2.11) becomes

$$
\begin{aligned}
\mathcal{T}^{\prime}: 0= & \left(1-\nu^{2}\right) x_{1}^{3}-\nu^{2} x_{2}^{3}-(1-\nu)^{2} x_{3}^{3}+\nu(1-3 \nu) x_{1}^{2} x_{2} \\
& -(1-\nu)(3+\nu) x_{1}^{2} x_{3}+\nu(1-3 \nu) x_{1} x_{2}^{2}-\nu(1-\nu) x_{2}^{2} x_{3} \\
& +(1-\nu)(3-\nu) x_{1} x_{3}^{2}+\nu(1-\nu) x_{2} x_{3}^{2}-2 \nu(1-\nu) x_{1} x_{2} x_{3} .
\end{aligned}
$$

Proof. The proof is analogous to the proofs of Lemmas 3.2 and 3.3.

Remark 3.7. The absolute invariants $(\rho, \sigma, \kappa)$ in equation (2.5) such that $\rho$ and $\kappa$ do not vanish simultaneously and $J_{10} \neq 0$ describe the moduli of genus-two curves with $J_{4} \cdot J_{6} \cdot J_{10} \neq 0$. The discussion of Lemma 3.6 proves that only for genus-two curves with $J_{4} \cdot J_{6} \cdot J_{10} \neq 0$, the conic $\mathcal{Q}$ in equation (2.8) is not guaranteed to have a rational point.

Substituting the parametrization of the conic $\mathcal{Q}^{\prime}$ in Lemma 3.3 into the cubic $\mathcal{T}^{\prime}$ in equation (3.7) and setting $U=x$ and $V=1$, one obtains the ramification locus of a sextic curve. The ramification locus is equivalent to $f(x)=\sum_{i=0}^{6} a_{i}(\alpha, \beta) x^{i}=0$, where we write the sextic polynomial in the form

$$
\begin{aligned}
f(x)= & \left(d_{0}^{(1)}+d_{0}^{(2)}\right) x^{6}+\left(d_{1}^{(1)}+d_{1}^{(2)}\right) \Lambda_{6} x^{5}+\left(d_{2}^{(1)}+d_{2}^{(2)}\right) \Lambda_{6} x^{4} \\
& +d_{3}^{(1)} \Lambda_{6}^{2} x^{3}+\left(d_{2}^{(1)}-d_{2}^{(2)}\right) \Lambda_{6}^{2} x^{2}+\left(d_{1}^{(1)}-d_{1}^{(2)}\right) \Lambda_{6}^{3} x+\left(d_{0}^{(1)}-d_{0}^{(2)}\right) \Lambda_{6}^{3} .
\end{aligned}
$$

In terms of the coordinates of the point $\left[x_{1}^{0}: x_{2}^{0}: x_{3}^{0}\right]$ in equation (3.5) we have set

$$
\begin{aligned}
d_{j}^{(1)}= & c_{j, 0}^{(1)}\left[\left(\rho^{2}+\gamma\right) x_{1}^{0}+2 \gamma \rho x_{2}^{0}\right] \gamma+3 c_{j, 1}^{(1)}\left[x_{1}^{0}+\rho x_{2}^{0}\right] \gamma \Lambda_{6}^{\delta_{j}} x_{3}^{0} \\
& -c_{j, 0}^{(1)}\left[\left(\rho^{2}+\gamma\right) x_{1}^{0}-2 \gamma \rho x_{2}^{0}\right] \rho^{2} \sigma^{-2} \Lambda_{6}\left(x_{3}^{0}\right)^{2}-3 c_{j, 1}^{(1)}\left[x_{1}^{0}-\rho x_{2}^{0}\right] \rho^{2} \sigma^{-2} \Lambda_{6}^{1+\delta_{j}}\left(x_{3}^{0}\right)^{3}
\end{aligned}
$$




$$
\begin{aligned}
& +\left[3 \sigma c_{j, 2}^{(1)}-2 \rho^{2} c_{j, 0}^{(1)}\right] \sigma^{-1} \Lambda_{6} x_{1}^{0}\left(x_{3}^{0}\right)^{2}+c_{j, 3}^{(1)} \Lambda_{6}^{1+\delta_{j}}\left(x_{3}^{0}\right)^{3}, \\
d_{j}^{(2)}= & c_{j, 0}^{(2)}\left[2 \rho x_{1}^{0}+\left(\rho^{2}+\gamma\right) x_{2}^{0}\right] \gamma^{2}+6 c_{j, 1}^{(2)}\left[\rho x_{1}^{0}+\gamma x_{2}^{0}\right] \gamma \Lambda_{6}^{\delta_{j}} x_{3}^{0} \\
& -c_{j, 0}^{(2)}\left[2 \gamma x_{1}^{0}-\rho\left(\rho^{2}+\gamma\right) x_{2}^{0}\right] \gamma \rho \sigma^{-2} \Lambda_{6}\left(x_{3}^{0}\right)^{2}-6 c_{j, 1}^{(2)}\left[x_{1}^{0}-\rho x_{2}^{0}\right] \gamma \rho \sigma^{-2} \Lambda_{6}^{1+\delta_{j}}\left(x_{3}^{0}\right)^{3} \\
& +c_{j, 2}^{(2)} \gamma \Lambda_{6}^{\delta_{j}} x_{1}^{0}\left(x_{3}^{0}\right)^{2} .
\end{aligned}
$$

All coefficients remain regular and in general non-vanishing for $\sigma=0$ since $x_{0}^{3} / \sigma=\beta$. Here, $(\alpha, \beta)$ is a pair solving equation (3.4), and the coefficients $c_{j, k}^{(n)}$ are given by

$$
\begin{array}{lll}
c_{0,0}^{(1)}=18 \gamma \Lambda_{1}+3 \gamma \Lambda_{3}+\Lambda_{1} \Lambda_{3}, & c_{0,1}^{(1)}=\gamma+\Lambda_{1}, \\
c_{0,2}^{(1)}=-\gamma \Lambda_{3}+\Lambda_{5}, & c_{0,3}^{(1)}=-4 \gamma+\Lambda_{1}, \\
c_{0,0}^{(2)}=27 \gamma \rho+\gamma \Lambda_{2}+3 \kappa \Lambda_{3}, & c_{0,1}^{(2)}=\kappa, \\
c_{0,2}^{(2)}=-\gamma \Lambda_{2}+3 \Lambda_{4}, & \\
c_{1,0}^{(1)}=6\left(\gamma+\Lambda_{1}\right), & c_{1,1}^{(1)}=2\left(18 \gamma \Lambda_{1}+\gamma \Lambda_{3}+\Lambda_{1} \Lambda_{3}+2 \Lambda_{5}\right), \\
c_{1,2}^{(1)}=2\left(-2 \gamma+3 \Lambda_{1}\right), & c_{1,3}^{(1)}=6\left(-\gamma \Lambda_{3}+\Lambda_{5}\right), \\
c_{1,0}^{(2)}=12 \kappa, & c_{1,1}^{(2)}=18 \gamma \rho+2 \kappa \Lambda_{3}+2 \Lambda_{4}, \\
c_{1,2}^{(2)}=12 \kappa \Lambda_{6}, & \\
c_{2,0}^{(1)}=3\left(18 \gamma \Lambda_{1}-\gamma \Lambda_{3}+\Lambda_{1} \Lambda_{3}+4 \Lambda_{5}\right), & c_{2,1}^{(1)}=5\left(-\gamma+3 \Lambda_{1}\right), \\
c_{2,2}^{(1)}=72 \gamma \Lambda_{1}+\gamma \Lambda_{3}+4 \Lambda_{1} \Lambda_{3}+11 \Lambda_{5}, & c_{2,3}^{(1)}=15 \Lambda_{1}, \\
c_{2,0}^{(2)}=3\left(9 \gamma \rho-\gamma \Lambda_{2}+\kappa \Lambda_{3}+4 \Lambda_{4}\right), & c_{2,1}^{(2)}=5 \kappa, \\
c_{2,2}^{(2)}=3\left(36 \gamma \rho+\gamma \Lambda_{2}+4 \kappa \Lambda_{3}+\Lambda_{4}\right), & \\
c_{3,0}^{(1)}=20\left(-\gamma+\Lambda_{1}\right), & & c_{3,1}^{(1)}=4\left(18 \gamma \Lambda_{1}-\gamma \Lambda_{3}+\Lambda_{1} \Lambda_{3}+4 \Lambda_{5}\right), \\
c_{3,2}^{(1)}=20 \Lambda_{1}, & c_{3,3}^{(1)}=4\left(36 \gamma \Lambda_{1}+3 \gamma \Lambda_{3}+2 \Lambda_{1} \Lambda_{3}+3 \Lambda_{5}\right) .
\end{array}
$$

The coefficients $\Lambda_{1}, \ldots, \Lambda_{5}$ and $\Lambda_{6}$ were given in equations (3.8) and (3.2), respectively.

Remark 3.8. Equation (3.10) allows to easily describe the change in the sextic polynomial under the action of the automorphism of the conic $\mathcal{Q}^{\prime}$ given by $\left[x_{1}: x_{2}: x_{3}\right] \mapsto\left[ \pm x_{1}: \pm x_{2}: x_{3}\right]$.

We make the following remarks:

Remark 3.9. The transformation $x \rightarrow \frac{\Lambda_{6}}{x}$ maps the coefficients $d_{k}^{(1)} \pm d_{k}^{(2)} \mapsto d_{k}^{(1)} \mp d_{k}^{(2)}$ for $k=0,1,2$ and $a_{3} \mapsto a_{3}$. This is to be expected since the coefficients are in terms of invariants of the binary sextic $f(x, z)$ and $x \rightarrow \frac{1}{x}$ just permutes $x$ and $z$.

Remark 3.10. The fact that the coefficients defining $\mathcal{C}_{(\alpha, \beta)}$ are polynomials of the new absolute invariants $\gamma, \kappa, \sigma$ and appear in the particular pattern given by equation (3.10) opens up the question about their meaning. It turns out that in the context of the F-theory/heterotic string theory duality the new invariants parameterize the physical defects of a certain class of $6 \mathrm{~d}$ $\mathcal{N}=(1,0)$ non-geometric vacua of the heterotic string when dualizing to F-theory. We will address this question in more detail in future work.

We have the following main result:

Theorem 3.11. Let $\mathfrak{p} \in \mathcal{M}_{2}$ such that $\mathfrak{p} \in \mathcal{M}_{2}(K)$, for some number field $K$, and $\mathfrak{j}=\left[J_{2}\right.$ : $\left.J_{4}: J_{6}: J_{10}\right]$ the corresponding point in $\mathbb{W P}_{(2,4,6,10)}^{3}\left(\mathcal{O}_{K}\right)$, where $\mathcal{O}_{K}$ is the ring of integers of $K$. A genus-two curve corresponding to $\mathfrak{p}$ is constructed as follows: 
i) If $J_{2} \cdot J_{10} \neq 0$ there is a genus-two curve $\mathcal{C}_{(\alpha, \beta)}$ given by

$$
\mathcal{C}_{(\alpha, \beta)}: y^{2}=\sum_{i=0}^{6} a_{i}(\alpha, \beta) x^{i},
$$

with coefficients given in equations (3.9) and (3.10), and a pair $(\alpha, \beta)$ satisfying

$$
\alpha^{2}+\Lambda_{6} \beta^{2} \sigma=\gamma
$$

where $\Lambda_{6}, \sigma$, and $\gamma$ are determined by $\mathfrak{p}$. Moreover, $\mathcal{C}_{(\alpha, \beta)}$ is defined over its field of moduli $K$, i.e., $a_{i}(\alpha, \beta) \in K, i=0, \ldots, 6$, if and only if $K$-rational $\alpha$ and $\beta$ exist.

ii) If $J_{2}=0$ and $J_{4} \cdot J_{6} \cdot J_{10} \neq 0$, there is a genus-two curve given by setting $\rho=\kappa \neq 0$ in equation (3.11).

iii) If $J_{2}=J_{6}=0$ and $J_{4} \cdot J_{10} \neq 0$, there is only one genus-two curve given by

$$
\begin{aligned}
y^{2}= & (4 \nu+1)(2 \nu-1) x^{6}+2(1-\nu)(4 \nu+3) x^{5}-15(1-\nu) x^{4} \\
& +20(1-\nu)^{2} x^{3}+5(2 \nu-3)(1-\nu)^{2} x^{2}+6(1-\nu)^{3} x-(1-\nu)^{3}
\end{aligned}
$$

with $\nu=J_{4}^{5} /\left(2^{2} 3^{5} 5^{5} J_{10}^{2}\right)$.

iv) If $J_{2}=J_{4}=0$ and $J_{6} \cdot J_{10} \neq 0$, there is only one genus-two curve given by

$$
\begin{aligned}
y^{2}= & 5 x^{6}+12(1-\mu) x^{5}-15(1-\mu) x^{4}-80(1-\mu)^{2} x^{3} \\
& +15(4 \mu-7)(1-\mu)^{2} x^{2}-60(1-\mu)^{3} x+(4 \mu-13)(1-\mu)^{3} .
\end{aligned}
$$

with $\mu=J_{6}^{5} /\left(2^{4} 3^{4} 5^{5} J_{10}^{3}\right)$.

v) If $J_{2}=J_{4}=J_{6}=0$ and $J_{10} \neq 0$, there is only one genus-two curve given by

$$
y^{2}=x^{6}-x .
$$

Proof. We already proved that there are genus-two curves $y^{2}=f(x)$ corresponding to $\mathfrak{p}$, where $f(x)$ is given in equation (3.9). We obtain coefficients $a_{i}(\alpha, \beta) \in \mathbb{Q}\left[\alpha, \beta, J_{2}, J_{4}, J_{6}, J_{10}\right]$ for $0 \leq i \leq 6$. The field of moduli $K$ of the point $\mathfrak{p}$ is $K=\mathbb{Q}\left(\mathbf{x}_{1}, \mathbf{x}_{2}, \mathbf{x}_{3}\right)$. For $J_{2} \neq 0$ the invariants $(\rho, \sigma, \kappa)$ are birationally equivalent to $\left(\mathbf{x}_{1}, \mathbf{x}_{2}, \mathbf{x}_{3}\right)$ over $\mathbb{Q}$ by Lemma 2.3. By Lemma 3.1 the conic $\mathcal{Q}$ in equation $(2.8)$ had a $K$-rational point if and only if the conic $\mathcal{Q}^{\prime}$ in equation (3.1) does. By Lemma 3.2 the conic $\mathcal{Q}^{\prime}$ has a $K$-rational point, i.e., there is a $K$ rational solution $(\alpha, \beta)$ of equation (3.4). Therefore, $a_{i}(\alpha, \beta) \in K$, for $i=0, \ldots, 6$. The cases with $J_{4} \cdot J_{6}=0$ are similarly obtained by applying Lemmas 2.3 and 3.6.

This completes the proof.

Remark 3.12. The four pairs $( \pm \alpha, \pm \beta)$ belong to the same conic $\mathcal{Q}^{\prime}$. Therefore, we get four genus-two curves in Theorem 3.11, but they are all twists of each other. That is, we get one curve (over the algebraic closure), but four twists.

The main benefit of the above result is that it will give a curve defined over $\mathbb{Q}$ whenever possible. This is an improvement from results in [18] where a curve is provided only for curves with automorphism group of order 2 and $J_{2} \neq 0$. The equation is valid even when the field of moduli is not a field of definition. Hence, for every point $\mathfrak{p} \in \mathcal{M}_{2}$ we get a curve. Next we have the following result: 
Corollary 3.13. For every point $\mathfrak{p} \in \mathcal{M}_{2}$ such that $\mathfrak{p} \in \mathcal{M}_{2}(K)$, for some number field $K$, there is a genus-two curves $\mathcal{C}$ given by

$$
\mathcal{C}_{(\alpha, 0)}: y^{2}=\sum_{i=0}^{6} a_{i}(\alpha, 0) x^{i}
$$

corresponding to $\mathfrak{p}$, such that $a_{i}(\alpha, 0) \in K(\alpha), i=0, \ldots, 6$ as given in equation (3.9). Moreover, $\mathcal{C}_{(\alpha, 0)}$ is at worst defined over the quadratic extension $K(\alpha)$ of the field of moduli $K$ with $\alpha^{2}=$ $\rho^{2}+\sigma$.

We have the immediate consequence:

Corollary 3.14. Let $\mathbf{x}_{1}, \mathbf{x}_{2}, \mathbf{x}_{3}$ be transcendentals. There exists a genus-two curve $\mathcal{C}_{(\alpha, 0)}$ defined over $\mathbb{Q}\left(\mathbf{x}_{1}, \mathbf{x}_{2}, \mathbf{x}_{3}\right)[\alpha]$ with $\alpha^{2}=\rho^{2}+\sigma$ such that

$$
\mathbf{x}_{1}\left(\mathcal{C}_{(\alpha, 0)}\right)=\mathbf{x}_{1}, \quad \mathbf{x}_{2}\left(\mathcal{C}_{(\alpha, 0)}\right)=\mathbf{x}_{2}, \quad \mathbf{x}_{3}\left(\mathcal{C}_{(\alpha, 0)}\right)=\mathbf{x}_{3}
$$

We have the following corollary:

Corollary 3.15. Let $\sigma=0$ and $\rho \neq 0$ for $\mathfrak{p} \in \mathcal{M}_{2}$. Then, there is a genus-two curve $\mathcal{C}$ given by Corollary 3.13, and it is defined over the field of moduli.

Proof. For $\sigma=0$ and $\rho \neq 0$, we have $\gamma=\rho^{2}$, and we choose the $K$-rational solution $(\alpha, \beta)=$ $(\rho, 0)$ in equation $(3.4)$.

Remark 3.16. It is easy to check using equation (2.5) that the locus $\sigma=0$ and $\rho \neq 0$ for $\mathfrak{p} \in \mathcal{M}_{2}$ corresponds to the locus

$$
J_{10}=-2^{-11} 3^{-3} 5^{-5}\left(9 J_{2}^{5}-700 J_{2}^{3} J_{4}+2400 J_{2}^{2} J_{6}-262400 J_{2} J_{4}^{2}+768000 J_{4} J_{6}\right) .
$$

We have the following lemma:

Lemma 3.17. In terms of the invariants $\rho, \sigma, \kappa$ and $\gamma=\rho^{2}+\sigma$, we have

$$
\begin{aligned}
& D=-\frac{J_{2}^{5}\left((\kappa-\rho)^{2}+9 \rho\right)\left((2 \kappa-\rho)^{2}-\gamma\right)}{2^{17} 3^{7} 5^{5}(\kappa-\rho)^{5}}, \\
& R^{2}=\frac{J_{2}^{15}\left((\kappa-\rho)^{2}+9 \rho\right)^{3} \Lambda_{6}}{2^{54} 3^{21} 5^{15}(\kappa-\rho)^{15}}
\end{aligned}
$$

In particular, the locus $D=0$ and $\chi_{35} \neq 0$ is given by $\gamma=(2 \kappa-\rho)^{2}$ or, equivalently, $\sigma=$ $4 \kappa(\kappa-\rho)$.

We have the following corollary:

Corollary 3.18. Let $D=0$ and $\chi_{35}^{2} \neq 0$ for $\mathfrak{p} \in \mathcal{M}_{2}$. Then, there is a genus-two curve $\mathcal{C}$ given by Corollary 3.13, and it is defined over the field of moduli.

Proof. For $\gamma=(2 \kappa-\rho)^{2}$ we can choose $(\alpha, \beta)=(\rho-2 \kappa, 0)$ in equation (3.4). As $\kappa-\rho \neq 0$ we have $y_{0} \neq 0$ in equation $(3.5)$. 


\subsection{A word about extra automorphisms}

In this section we derive a sextic polynomial for the sublocus of $\mathcal{M}_{2}$ with $\chi_{35}=0$. We have the following proposition:

Proposition 3.19. Let $D \neq 0$ and $\chi_{35}=0$ for $\mathfrak{p} \in \mathcal{M}_{2}$. Then, there is a genus-two curve $\mathcal{C}: y^{2}=F(x)$ with

$$
F(x)=\left(d_{0}^{(1)}+d_{0}^{(2)}\right) x^{6}+\left(d_{2}^{(1)}+d_{2}^{(2)}\right) x^{4}+\left(d_{2}^{(1)}-d_{2}^{(2)}\right) x^{2}+\left(d_{0}^{(1)}-d_{0}^{(2)}\right),
$$

and with coefficients in $\mathbb{Z}[\alpha, \rho, \kappa]$ given by

$$
\begin{aligned}
& d_{0}^{(1)}=3 \kappa \gamma^{2}-\left(\kappa^{2}+9 \rho\right)(11 \kappa-9 \rho-126) \gamma-\left(\kappa^{2}+9 \rho\right)^{2}(4 \kappa-3 \rho-36), \\
& d_{0}^{(2)}=\left(\gamma^{2}+\left(-\kappa^{2}+3 \kappa \rho+45 \rho\right) \gamma-3 \kappa\left(\kappa^{2}+9 \rho\right)(4 \kappa-3 \rho-36)\right) \alpha, \\
& d_{2}^{(1)}=-15 \kappa \gamma^{2}+15\left(\kappa^{2}+9 \rho\right)(5 \kappa-3 \rho-18) \gamma-15\left(\kappa^{2}+9 \rho\right)^{2}(4 \kappa-3 \rho-36), \\
& d_{2}^{(2)}=\left(-15 \gamma^{2}+\left(75 \kappa^{2}-45 \kappa \rho-135 \rho\right) \gamma-15 \kappa\left(\kappa^{2}+9 \rho\right)(4 \kappa-3 \rho-36)\right) \alpha .
\end{aligned}
$$

Here, the absolute invariants $\alpha, \gamma, \rho, \kappa$ are subject to the constraints $\Lambda_{6}=0$ in equation (3.2) and $\alpha^{2}=\gamma$.

Proof. For $\Lambda_{6}=\epsilon^{2}$ with $\epsilon \rightarrow 0$, we rescale the polynomial in equation (3.12) according to $f(\epsilon x) / \epsilon^{6}$ before setting $\epsilon=0$. If we substitute $\Lambda_{6}=0$ into equation (3.4) we obtain $\alpha^{2}=\gamma$, $\beta=0$. Therefore, we will use the absolute invariants $\alpha, \gamma, \rho, \kappa$ subject to the constraints $\Lambda_{6}=0$ in equation (3.2) and $\alpha^{2}=\gamma$. The sextic polynomial in equation (3.12) has coefficients in $\mathbb{Z}[\alpha, \rho, \kappa]$. The remainder of the proof then follows from specializing the formulas in equation (3.13) to $\beta=\Lambda_{6}=0$.

The polynomial in equation (3.12) is a twist of the polynomial given by

$$
\hat{F}(x)=x^{6}+a x^{4}+b x^{2}+1 .
$$

The curve $y^{2}=\hat{F}(x)$ has extra involutions, i.e., it has automorphisms other than the hyperelliptic involution, for appropriate values of $a, b$ (the discriminant is nonzero). In [19] for curves with automorphism the dihedral invariants

$$
u=a b, \quad v=a^{3}+b^{3},
$$

were defined which give a birational parametrization of this locus $\mathcal{L}_{2}$ which is a two-dimensional subvariety of $\mathcal{M}_{2}$. We have the following:

Corollary 3.20. For the genus-two curve $\mathcal{C}: y^{2}=F(x)$ given by equation (3.12) with $\chi_{35}=0$ we obtain the dihedral invariants

$$
\begin{aligned}
& u=\frac{\left(d_{2}^{(1)}+d_{2}^{(2)}\right)\left(d_{2}^{(1)}-d_{2}^{(2)}\right)}{\left(d_{0}^{(1)}+d_{0}^{(2)}\right)\left(d_{0}^{(1)}-d_{0}^{(2)}\right)}, \\
& v=\frac{\left(d_{2}^{(1)}-d_{2}^{(2)}\right)^{3}}{\left(d_{0}^{(1)}+d_{0}^{(2)}\right)\left(d_{0}^{(1)}-d_{0}^{(2)}\right)^{2}}+\frac{\left(d_{2}^{(1)}+d_{2}^{(2)}\right)^{3}}{\left(d_{0}^{(1)}+d_{0}^{(2)}\right)^{2}\left(d_{0}^{(1)}-d_{0}^{(2)}\right)},
\end{aligned}
$$

and the Igusa invariants $\left[J_{2}: J_{4}: J_{6}: J_{10}\right]$ given by [19, equation (16)]. 


\section{References}

[1] Beshaj L., Hidalgo R., Malmendier A., Kruk S., Quispe S., Shaska T., Rational points on the moduli space of genus two, in Algebraic Curves and their Fibrations in Mathematical Physics and Arithmetic Geometry, Contemporary Math., Vol. 703, Amer. Math. Soc., Providence, RI, 2018, 87-120.

[2] Bolza O., On binary sextics with linear transformations into themselves, Amer. J. Math. 10 (1887), 47-70.

[3] Booker A.R., Sijsling J., Sutherland A.V., Voight J., Yasaki D., A database of genus-2 curves over the rational numbers, LMS J. Comput. Math. 19 (2016), suppl. A, 235-254, arXiv:1602.03715.

[4] Bröker R., Howe E.W., Lauter K.E., Stevenhagen P., Genus-2 curves and Jacobians with a given number of points, LMS J. Comput. Math. 18 (2015), 170-197, arXiv:1403.6911.

[5] Clebsch A., Gordan P., Theorie der Abelschen Functionen, Thesaurus Mathematicae, Vol. 7, Physica-Verlag, Würzburg, 1967.

[6] Freitag E., Siegelsche Modulfunktionen, Grundlehren der Mathematischen Wissenschaften, Vol. 254, Springer-Verlag, Berlin, 1983.

[7] Goren E.Z., Lauter K.E., Genus 2 curves with complex multiplication, Int. Math. Res. Not. 2012 (2012), 1068-1142, arXiv:1003.4759.

[8] Gritsenko V.A., Nikulin V.V., Igusa modular forms and "the simplest" Lorentzian Kac-Moody algebras, Sb. Math. 187 (1996), 1601-1641.

[9] Harris J., Morrison I., Moduli of curves, Graduate Texts in Mathematics, Vol. 187, Springer-Verlag, New York, 1998.

[10] Igusa J.I., Arithmetic variety of moduli for genus two, Ann. of Math. 72 (1960), 612-649.

[11] Igusa J.I., On Siegel modular forms of genus two, Amer. J. Math. 84 (1962), 175-200.

[12] Igusa J.I., Modular forms and projective invariants, Amer. J. Math. 89 (1967), 817-855.

[13] Igusa J.I., On the ring of modular forms of degree two over Z, Amer. J. Math. 101 (1979), 149-183.

[14] Krishnamoorthy V., Shaska T., Völklein H., Invariants of binary forms, in Progress in Galois Theory, Dev. Math., Vol. 12, Springer, New York, 2005, 101-122, arXiv:1209.0446.

[15] Lauter K., Naehrig M., Yang T., Hilbert theta series and invariants of genus 2 curves, J. Number Theory 161 (2016), 146-174.

[16] Malmendier A., Morrison D.R., K3 surfaces, modular forms, and non-geometric heterotic compactifications, Lett. Math. Phys. 105 (2015), 1085-1118, arXiv:1406.4873.

[17] Malmendier A., Shaska T., The Satake sextic in F-theory, J. Geom. Phys. 120 (2017), 290-305, arXiv:1609.04341.

[18] Mestre J.F., Construction de courbes de genre 2 à partir de leurs modules, in Effective Methods in Algebraic Geometry (Castiglioncello, 1990), Progr. Math., Vol. 94, Birkhäuser Boston, Boston, MA, 1991, 313-334.

[19] Shaska T., Völklein H., Elliptic subfields and automorphisms of genus 2 function fields, in Algebra, Arithmetic and Geometry with Applications (West Lafayette, IN, 2000), Springer, Berlin, 2004, 703-723, math.AG/0107142. 